\title{
Characterising Fatigue Behaviour of Nodular Cast Iron Us- ing Micromechanical Simulations
}

\author{
Mehul Lukhi ${ }^{1, *}$, Meinhard Kuna $^{1}$, and Geralf Hütter ${ }^{1}$ \\ ${ }^{1}$ Institute of Mechanics and Fluid dynamics, TU Bergakademie Freiberg, Germany
}

\begin{abstract}
Elastic-plastic porous materials experience an increase in the mean void volume fraction when they are subjected to cyclic loading. This behavior is known both from the experiments and simulations in the literature. The authors have first time used this mechanism for the evaluation of the fatigue life in nodular cast iron. In this contribution, the stress-life approach is presented for the characterization of fatigue failure. For this purpose, micromechanical finiteelement simulations are carried out using the axisymmetric cell model. The cell model having isotropic/non-linear kinematic hardening behavior is subjected to fully reversed cyclic stress controlled loading. The finite element simulations are carried out cycle by cycle until the final failure of the cell model. The numbers of cycles to failure are extracted from the simulations. The stress-life curves are shown for spherical and elliptical graphite particle cell models. The results of the micromechanical simulations are in qualitative agreement with the typical experimental stress-life curves.
\end{abstract}

\section{Introduction}

Nodular cast iron (NCI) is an important ferrous material. It consists of graphite particles which are nodular in shape, and matrix. The matrix can be ferritic, pearlitic, austenitic etc. The type of matrix microstructure depends on the heat treatment of the NCI. NCI is different from the other types of cast iron in terms of the shape of the graphite particle. The nodular shape is promoted by adding magnesium or cerium to the liquid iron. The nodular shape of graphite particle improves the mechanical properties like strength and toughness of cast iron. NCI has comparable mechanical properties like engineering steels at the reduced manufacturing costs which makes it very popular in different applications like gearboxes, crankshafts, wind turbine rotor hubs, nuclear storage and transportation casks etc [1].

The fatigue of materials is a very important topic from the economic, engineering and safety point of view. The metal fatigue leads to failure of structures and components. To prevent fatigue failure, it is important to understand fatigue failure mechanisms. Many researchers have provided insightful information regarding fatigue in NCI from the experiments like Murakami [2], Fischer et al.[3], Cavallini et al.[4], Greno et al.[5], Asi [6], Xue et al.[7], Komotori et al.[8] to name a few. The small defects, matrix, and microshrinkages have major influence on the fatigue strength of NCI[9]. With comparison of steels with similar matrix properties, NCI has lower fatigue strength due to presence of graphite nodules and other microstructrual defects. According to Endo[9], the largest graphite nodule and the

\footnotetext{
*e-mail: mehul.lukhi@imfd.tu-freiberg.de
} 
largest microshrikages are harmful defects for NCI with ferritic matrix. Using the $\sqrt{\text { area }}$ of the largest defect parameter model, the fatigue limit can be predicted [9]. The edurance limit can be related to a crack, initiated from graphite nodules or casting defects propagation in NCI and microshrinkage pores act as cites for the fatal crack propagating at the endurance limit [10]. Costa et al. [11] has suggested that casting defects, graphite nodules as defects, the distribution of defects in a area near the surface should be used as input parameters for the model to predict fatigue life in NCI. The rotating bending fatigue limit reduces $30 \%$ for the thick walled castings compared to thin walled castings of NCI[12]. This is caused by longer solidification time. It leads to an overgrowth of the graphite nodules and chunky graphite. It shows that not only the largest defects are not only involved in the fatigue damage, as also the graphite shape also has major influence on the fatigue damage of NCI. Kevian Kasavayee has mentioned that the fracture surface of NCI after cyclic loading can show the following features[13].

- voids related to the decohesion of the graphite particle from the matrix, similar to to microvoid growth relating to dimple formation

- secondary cracks in the graphite particles

- intergranular and transgranular fractures, with or without striation marks

In this study, the authors have explored the idea that voids generated due to decohesion are growing with cyclic loading. An increase in the mean volume fraction of voids with every cycle is called void ratchetting. The authors have considered void ratchetting as a failure mechanism of low cycle fatigue (LCF) and extremely low cycle fatigue (ELCF) in NCI. Fatigue life can be characterized by the stress-life approach, the strain-life approach and the fracture mechanics-based approach. The authors have previously studied the strainlife approach using the micromechanical simulations and the results of this approach have been published [14].

This contribution is organised as following. The introduction is followed by the stresslife section. In this section, the cell model and methodology used for the stress-life method are presented. The simulation results of the stress-life approach are also presented. The macroscopic hysteresis loops and the macroscopic response of the model are mentioned. The stress-life curves prepared from the simulation results are shown. This paper is concluded with the summary and outlook.

\section{Stress-life approach}

To investigate the stress-life approach, micromechanical simulations are carried out on the axisymmetric cell model shown in Fig. 1 using the commercial finite element software Abaqus.

\subsection{Cell model}

NCI microstructure consists of matrix and graphite nodules which can be idealized as hexagonal cells with graphite nodules at the body center position. It is assumed that the hexagonal structure can be replaced by a cylindrical one for the sake of simplicity. Now using the rotational symmetry, only the quarter part of the cylinder is modeled for the finite-element formulation. The finite element model prepared for the stress-life approach is an axisymmetric one. The graphite particle can be modeled as a void or as a rigid particle. In this study, the graphite is modelled as a rigid particle. The size of the graphite particle can be determined using the volume fraction of the graphite in the NCI. The shape of the graphite particle can be quantified using the shape factor $(S)$ which is defined in Eq. (1). The volume fraction of the 
graphite particle in cell model is defined according to Eq. (2). Here, the considered volume fraction of graphite is $11 \%$.

$$
\begin{gathered}
S=\frac{4 \pi A}{U^{2}} \\
f_{0}=\frac{2 a_{0}^{2} b_{0}}{3 R_{0}^{2} L_{0}}
\end{gathered}
$$
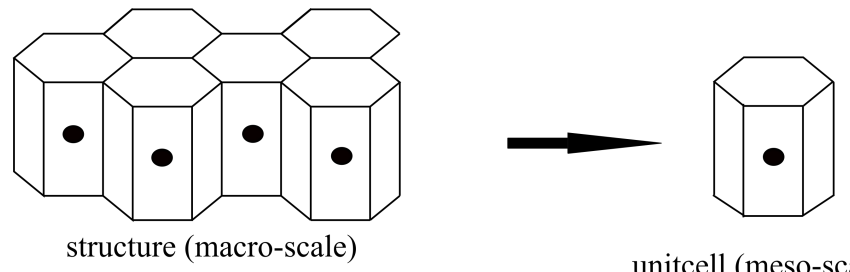

unitcell (meso-scale)
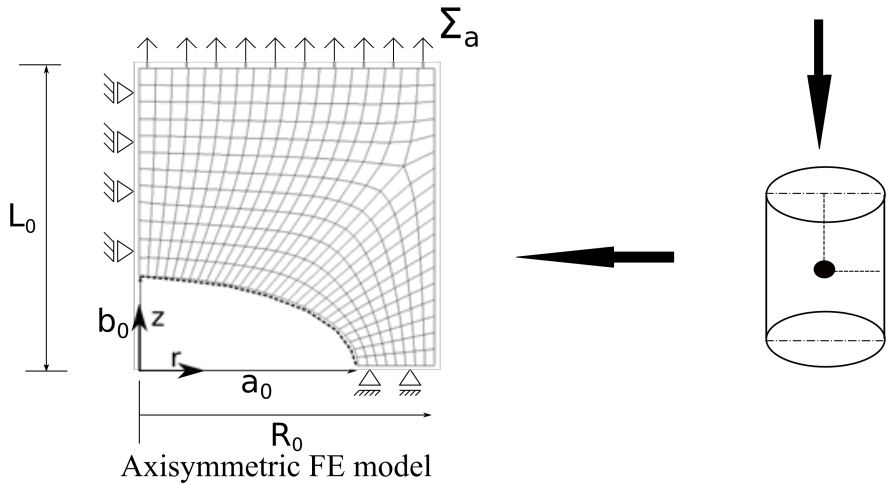

Figure 1: An axisymmetric cell model for the stress-life approach

The boundary conditions are applied according to the axisymmetric conditions $\left(u_{z}=0\right.$ at bottom surface and $u_{r}=0$ at left side surface of the cell model). The additional kinematic constraints are applied on the top and right surfaces in order to keep them plane. The cyclic surface traction is applied on the top surface. The dashed line in Fig. 1 represents the rigid line for the modeling of the graphite as a rigid particle. The surface to surface interaction is defined between graphite and matrix. The contact behavior consists of frictionless contact in the tangential direction and hard contact in the normal direction.

The discretization of the model is done using axisymmetric elements with full integration scheme (CAX4) in commercial FE-software Abaqus. This model is inspired by the previous work of the authors for the strain-life approach[14]. An isotropic/non-linear kinematic hardening model is used to describe the material behavior of the metallic matrix during the analysis. The yield function, the equation to evaluate yield stress and evolution equation for the back stress are mentioned below [15].

$$
\begin{gathered}
\phi=\sqrt{\frac{3}{2}\left(\boldsymbol{\sigma}^{\mathrm{dev}}-\boldsymbol{\alpha}\right):\left(\boldsymbol{\sigma}^{\mathrm{dev}}-\boldsymbol{\alpha}\right)}-\sigma_{\mathrm{y}} \\
\sigma_{\mathrm{y}}=\sigma_{0}+Q_{\infty}\left(1-\exp \left(-b \bar{\epsilon}^{\mathrm{pl}}\right)\right) \\
\dot{\boldsymbol{\alpha}}=\frac{1}{\sigma_{\mathrm{y}}} C \dot{\bar{\epsilon}}^{\mathrm{pl}}\left(\boldsymbol{\sigma}^{\mathrm{dev}}-\boldsymbol{\alpha}\right)-\gamma \boldsymbol{\alpha} \dot{\bar{\epsilon}}^{\mathrm{pl}}
\end{gathered}
$$


Where $\boldsymbol{\sigma}^{\mathrm{dev}}$ is the deviatoric part of the stress tensor, $\boldsymbol{\alpha}$ is the back stress tensor. $\sigma_{\mathrm{y}}$ is the yield stress which evolves with plastic strain accumulation. $\sigma_{0}$ is the initial yield stress. The parameters $Q_{\infty}$ and $b$ are related to Voce's law for hardening. $Q_{\infty}$ determines the maximum change allowed in the yield stress until it reaches the saturation value and $b$ is the rate of the saturation. The parameters $C$ and $\gamma$ are non-linear kinematic hardening parameters. $C$ is the kinematic hardening modulus and $\gamma$ is part of the recall term. The values of these parameters are obtained from the data received from the private communication with Prof. Peter Hübner from Mittweida, Germany. The experiments were performed on a ferritic steel grade, which has similar mechanical properties and microstructure like NCI with a ferritic matrix. The different parameter values are shown in Table 1.

\subsection{Methodology}

The model shown in Fig.1 is subjected to cyclic macroscopic stress $\Sigma_{a}$ at the top surface and simulations are run cycle by cycle till the failure of the model. The macroscopic response of the model is shown in Fig. 2. The macroscopic response for the applied macro stress amplitude $\left(\Sigma_{a}\right)=300 \mathrm{MPa}$ is shown in Fig. 2. The stress ratio, $R=\frac{\Sigma_{\min }}{\Sigma_{\max }}$ is -1 . The macroscopic strain is calculated according to Eq. (6).

$$
E_{z}(\%)=\frac{u_{z}}{L_{0}} \times 100
$$

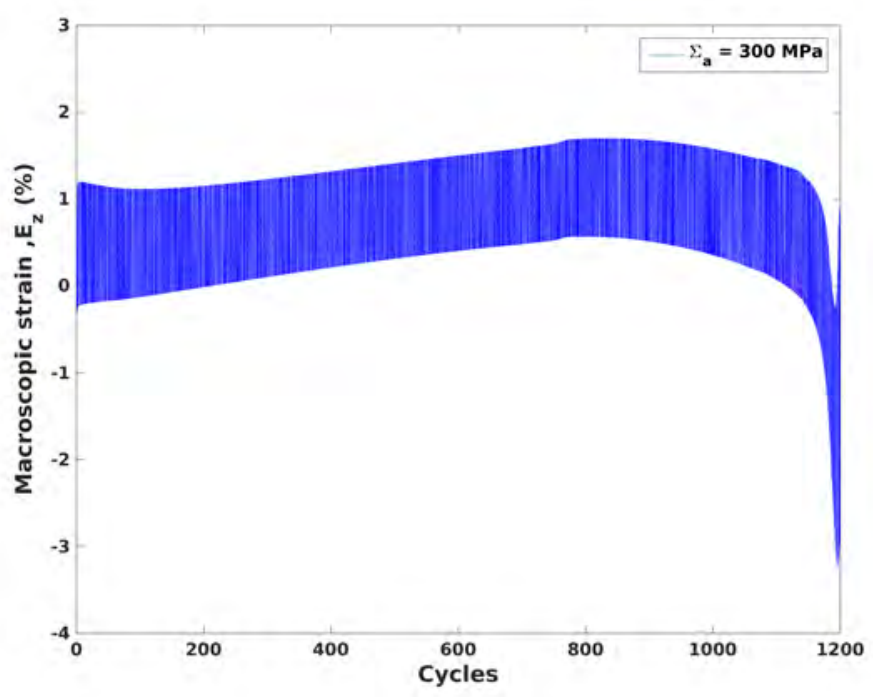

Figure 2: The macroscopic response (strain) of the axisymmetric cell model for the stress.life approach

There, $u_{z}$ is the displacement recorded at the reference point and $L_{0}$ is the length in the vertical direction as shown in Fig. 1 . The $E_{z}$ is gradually increasing and then it takes a nose dive. The physical interpretation of this behavior can be explained by the void ratchetting mechanism. The volume of the void, generated due to decohesion of matrix and the graphite increases cycles by cycle and the ligament is becoming shorter with an increase in the volume of the 
voids. When the ligament between the cells collapses it causes the coalescence of the voids. This leads to the sudden drop in $E_{z}$, which is defined as macroscopic failure in the present study.

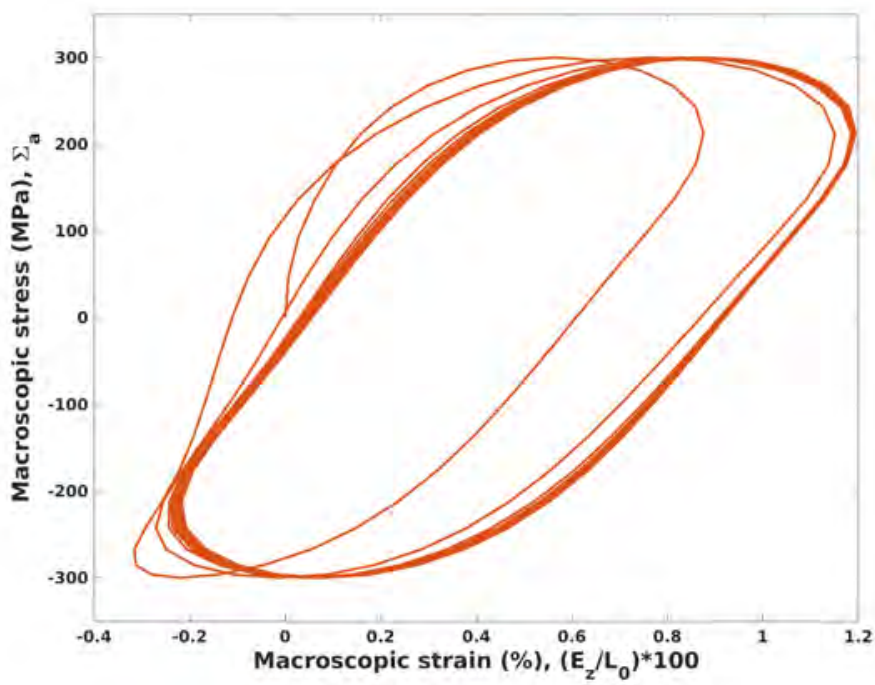

Figure 3: The macroscopic response (strain) of the axisymmetric cell model for the stress-life approach

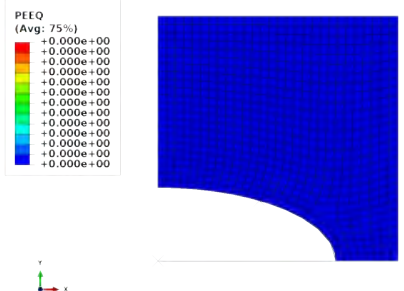

(a) At the beginning of the simulation

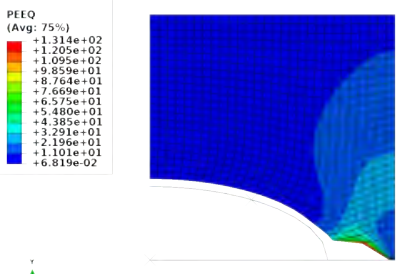

(b) At the end of the simulation

Figure 4: The void ratchetting mechanism with PEEQ distribution

The macroscopic stress-strain hysteresis loops are shown in Fig.3. Only the hysteresis loops for the initial 25 cycles are only shown Fig. 3. In Fig. 3, ordinate represents the applied macroscopic stress $\left(\Sigma_{a}\right)$ and abscissa represents the macroscopic strain calculated using Eq. (6). The finite element meshes at the beginning of the simulation and at the end of the simulation are shown with PEEQ distribution in Fig. 4. Fig. 4 (a) shows perfect mesh without any deformation. As the simulation progresses, the mesh undergoes deformation cycle by cycle. Fig. 4 (b) shows completely deformed ligament and deformed mesh. This is the mesh condition at the failure point. This point corresponds to the nosedive point in Fig. 2 . 
Using this method, the different stress amplitudes are applied to the cell model and simulations are carried out until the final failure of the model. The numbers of cycles to failure are extracted from the simulations.

\subsection{S-N curves}

The stress amplitude $\left(\Sigma_{a}\right)$ vs the number of cycles to failure $\left(N_{f}\right)$ are plotted on a log-log scale in Fig. 5. There are two S-N curves in Fig. 5. The full line represents the S-N curve for the model with spherical graphite particle $(S=1)$ and the dashed line represents the S-N curve for the cell model with elliptical graphite particle $(S=0.70)$. It is evident from Fig. 5 that the model with elliptical particle has lower fatigue life compared to the model with spherical particle.

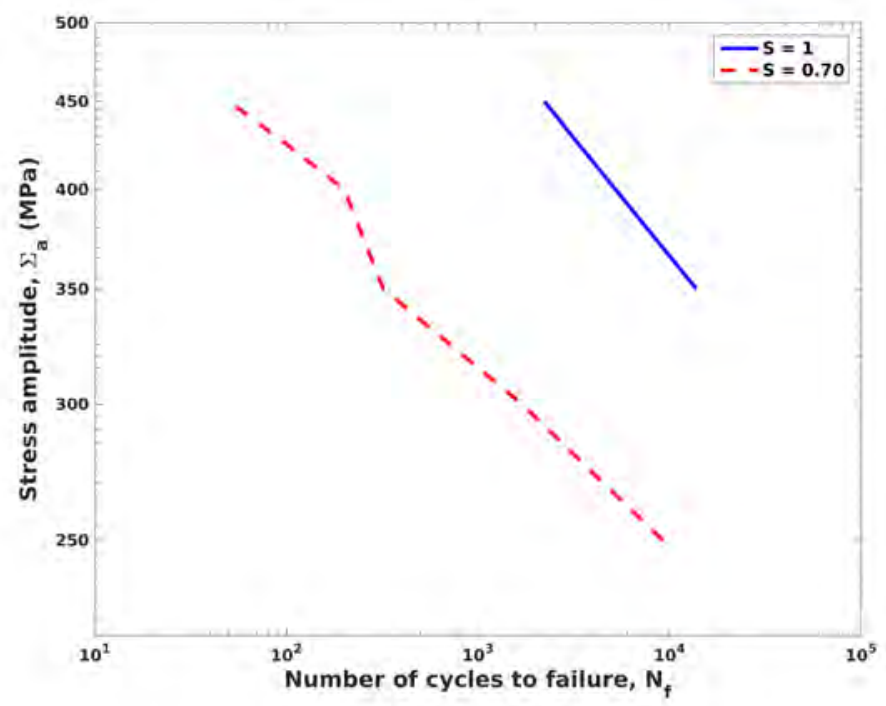

Figure 5: S-N curve prepared from the simulation results for $\mathrm{R}=-1$

For the purpose of comparison, strain-controlled simulations are carried out using the micromechanical cell model for the strain-life approach described in Lukhi et al. [14]. The cell model exhibits the void ratchetting and the sudden drop in the macroscopic stress is defined as the failure of the cell model. The simulations for different strain amplitudes have been performed and the strain-life curves have been prepared. In Fig. 6, the strain-life curves obtained from the simulations for the shape factor $S=0.70$ are shown together with the experimental data. The two different curves corresponds to graphite particle either modeled as a void or a rigid. From Fig. 6, it can be seen that the strain-life curve obtained from the simulation are in qualitative and quantitative agreement with the experiments. If the graphite particle modelled as a rigid, then the fatigue life reduces compared to the graphite particle modelled as a void.

From the comparison of the stress-life and strain-life approaches, the similarity in the drop of macroscopic responses is observed. The onset of void coalescence causes the nosedive in macroscopic strain and stress for the stress-life approach and the strain-life approach respectively. The dominating elastic deformation is observed in the finite element simulation of the stress-life approach, whereas the plastic deformation is dominating in case of the 
strain-life approach. From the simulation results, it is concluded that both approaches are complementary to each other. For the stress-life approach, stress amplitudes lower than 250 $\mathrm{MPa}$ are being investigated using the axisymmetric cell model.

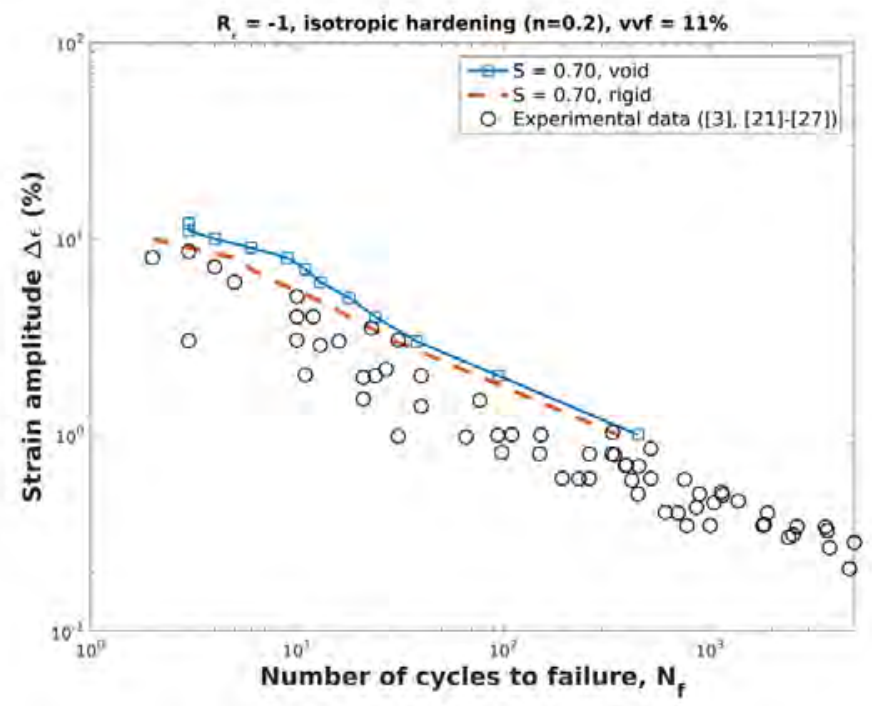

Figure 6: Strain life curves for strain ratio $R_{\epsilon}=-1$ [14]

Table 1: Material parameter for EN-GJS-400

\begin{tabular}{|l|l|}
\hline Parameters & Value \\
\hline $\mathrm{E}$ & $210000 \mathrm{MPa}$ \\
\hline$v$ & 0.3 \\
\hline$\sigma_{0}$ & $230 \mathrm{MPa}$ \\
\hline$Q_{\infty}$ & $105 \mathrm{MPa}$ \\
\hline $\mathrm{b}$ & 0.25 \\
\hline $\mathrm{C}$ & $78300 \mathrm{MPa}$ \\
\hline$\gamma$ & 105 \\
\hline
\end{tabular}

\section{Summary and Outlook}

In this study, the stress-life approach for the fatigue life evaluation is studied using micromechanical simulations. For this, an axisymmetric cell model is prepared. This model is subjected to fully reversed cyclic traction loading and cycle by cycle simulations are carried out until final failure of the cell model. The material behaviour is an elastic-plastic one with combined hardening. The cell model simulations are carried out for the spherical and the elliptical graphite particle are carried out and the results are plotted on the log-log curve. The results obtained from the simulations are in qualitative agreement with the expected stresslife curve. The stress-life curves from the experiments will be obtained from the literature and will be compared with the results numerical the simulations. Further on, we will study the effect of load ratio, matrix hardening (isotropic hardening and combined hardening), applied stress type(nominal or true) and graphite particle modeling as a void or rigid on the stress-life behavior using numerical experiments using the axisymmetric model. 


\section{Acknowledgement}

This work is funded by ESF (European social fund) scholarship for Mehul Lukhi under country innovation project (SAB application number: 100284311) in Saxony, Germany. The authors would also like to thank Prof. Peter Hübner for valuable discussions and providing the experimental data.

\section{References}

[1] G. Hütter, L. Zybell, M. Kuna, Engineering Fracture Mechanics 144, 118 (2015)

[2] Y. Murakami, in Metal Fatigue, edited by Y. Murakami (Elsevier Science Ltd, Oxford, 2002), pp. 205 - 216, ISBN 978-0-08-044064-4

[3] G. Fischer, J. Nellesen, N. Anar, K. Ehrig, H. Riesemeier, W. Tillmann, Materials Science and Engineering: A 577, 202 (2013)

[4] M. Cavallini, O. Di Bartolomeo, F. Iacoviello, Engineering Fracture Mechanics 75, 694 (2008)

[5] G. Greno, J. Otegui, R. Boeri, International Journal of Fatigue 21, 35 (1999)

[6] O. Asi, Engineering Failure Analysis 13, 1260 (2006)

[7] H. Xue, E. Bayraktar, C. Bathias, Journal of Materials Processing Technology 202, 216 (2008)

[8] J. Komotori, M. Shimizu, in Proceedings of The 7th International Conference On Fracture (ICF7), edited by K. Salama, K. Ravi-chandar, D. Taplin, P.R. Rao (Pergamon, Oxford, 1989), pp. 1213 - 1220, ISBN 978-0-08-034341-9

[9] M. Endo, Fatigue strength prediction of ductile irons subjected to combined loading, in ECF13, San Sebastian (2000)

[10] P. Clement, J.P. Angeli, A. Pineau, Fatigue \& Fracture of Engineering Materials \& Structures 7, 251 (1984)

[11] N. Costa, N. Machado, F. Silva, International Journal of Fatigue 32, 988 (2010)

[12] M. Benedetti, E. Torresani, V. Fontanari, D. Lusuardi, Metals 7, 88 (2017)

[13] K.A. Kasvayee, Ph.D. thesis, Jönköping University, Materials and Manufacturing, Research area Materials and manufacturing - Casting (2017)

[14] M. Lukhi, M. Kuna, G. Hütter, International Journal of Fatigue 113, 290 (2018)

[15] F. Rabold, M. Kuna, Computational Materials Science 32, 489 (2005), iWCMM 